\title{
EFFICIENT MANAGEMENT OF NON REDUNDANT RULES IN LARGE PATTERN BASES: A BITMAP APPROACH
}

\author{
François Jacquenet \\ Christine Largeron \\ Cédric Udréa \\ EURISE - University of Saint-Etienne \\ 23 rue Paul Michelon, F42023 Saint-Etienne, France \\ Francois.Jacquenet@univ-st-etienne.fr
}

Keywords: Pattern Management, Association Rules, Non-redundant Rules, Bitmap Arrays.

\begin{abstract}
Knowledge Discovery from Databases has more and more impact nowadays and various tools are now available to extract efficiently (in time and memory space) some knowledge from huge databases. Nevertheless, those systems generally produce some large pattern bases and then the management of these one rapidly becomes untractable.

Few works have focused on pattern base management systems and researches on that domain are really new. This paper comes within that context, dealing with a particular class of patterns that is association rules. More precisely, we present the way we have efficiently implemented the search for non redundant rules thanks to a representation of rules in the form of bitmap arrays. Some experiments show that the use of this technique increases dramatically the gain in time and space, allowing us to manage large pattern bases.
\end{abstract}

\section{INTRODUCTION}

Data mining, and more generally knowledge discovery from databases, have really been an active research area for the last decade. Volumes of data that have to be processed are larger and larger and data mining techniques are now varied and sophisticated. Various researches aimed at designing more and more efficient algorithms - in term of processing time and memory space - to mine huge volumes of data and commercial tools are now available for end user data miner. Nevertheless, one of the main drawbacks of these tools concerns the post processing step. Indeed, when a data miner uses a data mining tool on some data, he generally has no problem to parameterize it to his specific needs, but he often has some difficulty to interpret the results returned by this tool. In fact, what generally makes this interpretation difficult is that he is often overwhelmed by the quantity of patterns discovered by his data mining tool.

We may distinguish two principal ways for solving this problem. The first one consists in trying, during the data mining step, to avoid the generation of a too large quantity of patterns, keeping only the patterns that have an immediate interest for the user. This approach has been followed in several ways. For example, some algorithms only work on condensed representations of the patterns during the mining step (Boulicaut, 2005), (Zaki and Hsiao, 2005), (Wang et al., 2005), (Wang and Han, 2004), (Pasquier et al., 2005). Some algorithms push constraints in the frequent pattern generation step in order to immediately prune non relevant patterns during this phase (Bayardo et al., 2000), (Zaki, 2001), (Garofalakis et al., 2002), (Albert-Lorincz and Boulicaut, 2003), (Boulicaut and Jeudy, 2005). Nevertheless, even with such techniques the users generally remain overwhelmed with a large volume of patterns. Other algorithms try to integrate various sophisticated interestingness measure (Freitas, 1999) to only discover the patterns that seems to be interesting for the user, but this concept of interestingness of patterns is difficult to define a priori. Moreover, a user may want to process several data mining steps, choosing various parameterizations of his tool depending on the experiments he is doing, and then want to manage the various sets of patterns extracted.

Thus a second way, post-processing oriented, has focused on pattern base management. The principle consists in storing the patterns extracted by some data mining systems using some efficient data structures. Pattern manipulation languages have then to be designed in order to manage them. Data mining languages integrated in database management systems 
(Boulicaut and Masson, 2005) offer some facilities for pattern manipulation through post-processing operators. Nevertheless those one are very basic and pattern base management systems should provide more sophisticated functionalities. The PANDA project (Catania et al., 2004) is an interesting way in that domain. It proposes a generic framework to model various classes of patterns, then some SQL requests allow the user to manage those pattern bases. Nevertheless, as the underlying model used for storing the patterns is the relational model, the requests one can write are very complex, non intuitive and time consuming. Also in the field of pattern base management, we may cite the PMML project (Grossman et al., 1999) that allow interoperability of pattern bases, specifying an XML framework associated to the concept of pattern and providing some facilities for managing them in that form. (Zaki et al., 2005) also proposed a generic framework for defining data structures and management functionalities on patterns.

Our work is also part of this strategy that aims at designing data structures and efficient algorithms for large pattern base management. Indeed, we think that it may be interesting for the users, to be able to get all the patterns that may be extracted successively running data mining algorithms on various databases and then to have efficient tools for post processing them. More precisely, in this paper, we focus on the management of a particular class of patterns: the association rules (Agrawal et al., 1993). In this domain, few works have been done nowadays. The most significant one is probably RULE-QL (Tuzhilin and Liu, 2002) which proposes an extension of SQL allowing some kind of management for association rules. Nevertheless, such a language only offers some very basic functionalities such as accessing some parts of rules, searching for rules containing a particular item in their left or right part, etc. In fact, on such patterns, various management functions may be proposed and we focus more particularly on the discovery of non redundant rules in a set of association rules. This task has already been studied in several researches such as, for example, (Zaki, 2000), (Zaki, 2004), (Bastide et al., 2000), (Li et al., 2004), (Li and Hamilton, 2004), (Goethals et al., 2005). Nevertheless, all these works aim at defining data mining algorithms for extracting, from large databases, the non redundant rules, and only these one. Our work is different in that sense that it is based on a post processing approach, dealing with patterns and not directly data. Thus, we want, from a set of patterns (association rules), being able to extract all the non redundant one.

The next section recalls some basic definitions useful for understanding the paper. The section 3 presents some data structures that can be used for storing association rules. We first present a basic ap- proach and then another one based on bitmap arrays. The section 4 shows how one can take advantage of this former structure to evaluate the non redundancy of one rule with respect to another one. The section 5 presents an algorithm for extracting all the non redundant rules from a large rule base. A set of experiments shows the efficiency of our approach based on bitmap arrays with respect to the more basic one.

\section{DEFINITIONS}

Let us consider a database $\delta \subseteq I \times T$ where $I=$ $\{1,2, \ldots, k\}$ is an itemset, $T=\{1,2, \ldots, l\}$ is a set of transactions and such that if an item $i$ from $I$ is in a transaction $t$ from $T$, then $(\mathrm{i}, \mathrm{t}) \in \delta$. A set $X \subseteq I$ is called an itemset and its support, noted $|X|$, is equal to the number of transactions containing $X$.

\subsection{Association rules}

An association rule is an expression of the form $B \rightarrow H$ where $B=\left\{B_{1}, \ldots, B_{n}\right\}$ and $H=\left\{H_{1}, \ldots, H_{m}\right\}$ are some itemsets such that $\left\{B_{1}, \ldots, B_{n}\right\} \bigcap\left\{H_{1}, \ldots, H_{m}\right\}=\emptyset$. Each rule may be characterized by its support and confidence.

\section{Support of an association rule}

The support of an association rule of the form $B \rightarrow$ $H$ is equal to the number of transactions that contain the itemsets $B$ and $H$ divided by the total number of transactions in the database:

$$
\operatorname{Support}(B \rightarrow H)=\frac{|B H|}{l}
$$

where $l$ is the number of transactions in the database and $|B H|$ is the number of transactions in the database that contain the itemsets $B$ and $H$.

\section{Confidence of an association rule}

The confidence of an association rule of the form $B \rightarrow H$ is equal to the number of transactions of the database that contain the itemsets $B$ and $H$ divided by the number of transactions that contain the itemset $B$ :

$$
\operatorname{Confidence}(B \rightarrow H)=\frac{|B H|}{|B|}
$$

\subsection{Non redundant association rules}

Many definitions have been proposed in the literature concerning the concept of non redundant rules. For example, (Bastide et al., 2000) propose the following definition.

Definition: An association rule of the form $B \rightarrow H$ is non redundant if there exists no rule of the form 
$B^{\prime} \rightarrow H^{\prime}$ with the same support and confidence such that $B^{\prime} \subseteq B$ et $H \subseteq H^{\prime}$.

Many other definitions have been proposed, we may cite for example (Aggarwal and Yu, 1998), (Zaki, 2000), (Goethals et al., 2005), or the close definition of basic rule in (Li and Hamilton, 2004). In order to make the paper clearer, we will use, in the remaining of the paper, the following definition:

Definition: A rule of the form $B \rightarrow H$ is non redundant if and only if there exists no rule of the form $B^{\prime} \rightarrow H^{\prime}$ such that $B^{\prime} \subseteq B$ and $H \subseteq H^{\prime}$.

Nevertheless, our work could easily be extended to be based on the full definition of (Bastide et al., 2000) taking into account the support and confidence, but also the various other definitions.

\section{STORING ASSOCIATION RULES}

Before presenting an efficient algorithm for discovering non redundant rules in a large rulebase, it is essential to define an efficient data structure for storing those rules.

\subsection{Basic storage of association rules}

The most basic storage technique that can be used to store some association rule consists in using a single table, we call RULES, having three attributes:

- idrule: the identifier of the rule.

- idpart: the identifier of the part (1 for the left part of the rule, 2 for the right one).

- iditem: the identifier of the item.

Example: Let us consider $I=\{\mathrm{A}, \mathrm{B}, \mathrm{C}, \mathrm{D}, \mathrm{E}, \mathrm{F}\}$, and the rules $R=\{A, C\} \rightarrow\{D, E\}$ and $R^{\prime}=\{A\} \rightarrow$ $\{B, D, E\}$. The table RULES contains the following information:

\begin{tabular}{|c|c|c|}
\hline idrule & idpart & iditem \\
\hline \hline 1 & 1 & $\mathrm{~A}$ \\
1 & 1 & $\mathrm{C}$ \\
1 & 2 & $\mathrm{D}$ \\
1 & 2 & $\mathrm{E}$ \\
2 & 1 & $\mathrm{~A}$ \\
2 & 2 & $\mathrm{~B}$ \\
2 & 2 & $\mathrm{D}$ \\
2 & 2 & $\mathrm{E}$ \\
\hline
\end{tabular}

With this storage technique, the table contains as much rows as the number of rules multiplied by the number of items per rule. It is generally too large to be stored in main memory by the DBMS, which slows down the processing time due to the large number of hard disk accesses. This is the reason why we used another storage technique based on bitmap arrays.

\subsection{Storing rules with bitmap arrays}

(Morzy and Zakrzewicz, 1998) proposed the concept of bitmap arrays for storing association rules. With this approach, each part of an association rule contains as many bits as the total number of items in the database of transactions. Each bit of the bitmap array is then associated to a particular item and the value of this bit is equal to ' 1 ' if and only if the corresponding item appears in the part of the rule associated to the bitmap array. The binary coding of bitmap arrays reduces the storage cost and avoids access to data in order to count the number of items in a rule or in the result of a logical operation. Thus, as we will see later in the experimental section, using bitmap arrays dramatically reduces the processing time while searching for non redundant rules in a large rulebase.

Many works have used this representation of the rules in the framework of mining frequent patterns in order to optimize the algorithm designed. For example we may cite (Masson et al., 2004), (Pucheral et al., 1998), (Louie and Lin, 2000), etc.

In such a context, an association rule may then be stored in a table that contains two attributes: LeftPart and RightPart. Those attributes are bitmap arrays whose size is equal to the number of items of $I$, the set of all the items in the database of transactions.

Example: Let us consider $I=\{\mathrm{A}, \mathrm{B}, \mathrm{C}, \mathrm{D}, \mathrm{E}, \mathrm{F}\}$. The rule $R=\{A, C\} \rightarrow\{D, E\}$ is stored using the two following arrays:

Array LeftPart of R:

\begin{tabular}{|c|c|c|c|c|c|c|}
\hline Item & $\mathrm{F}$ & $\mathrm{E}$ & $\mathrm{D}$ & $\mathrm{C}$ & $\mathrm{B}$ & $\mathrm{A}$ \\
\hline Bit value & 0 & 0 & 0 & 1 & 0 & 1 \\
\hline
\end{tabular}

Array RightPart of R:

\begin{tabular}{|c|c|c|c|c|c|c|}
\hline Item & $\mathrm{F}$ & $\mathrm{E}$ & $\mathrm{D}$ & $\mathrm{C}$ & $\mathrm{B}$ & $\mathrm{A}$ \\
\hline Bit value & 0 & 1 & 1 & 0 & 0 & 0 \\
\hline
\end{tabular}

In the same way, the rule $R^{\prime}=\{A\} \rightarrow\{B, D, E\}$ is represented by the two following arrays:

Array LeftPart of R'

\begin{tabular}{|c|c|c|c|c|c|c|}
\hline Item & F & E & D & C & B & A \\
\hline Bit value & 0 & 0 & 0 & 0 & 0 & 1 \\
\hline
\end{tabular}

Array RightPart of R'

\begin{tabular}{|c|c|c|c|c|c|c|}
\hline Item & F & E & D & C & B & A \\
\hline Bit value & 0 & 1 & 1 & 0 & 1 & 0 \\
\hline
\end{tabular}

At the same time, all the name of the tables associated to each rule are stored in a global table $C O L$ $L E C T I O N$ which contains as much rows as the number of rules. This approach has several advantages. First, each rule is stored independently from the others. Secondly, the table COLLECTION is the only one that can become too large and make the system 
down. Nevertheless, the size of this table only depends on the number of rules and not of the number of items per rule contrary to the table RULES in the classical storage technique. Finally, if we have to manage several sets of association rules, it is possible to create several tables COLLECTION, which avoids to duplicate the rules contained in several distinct sets.

\subsection{Using the logical operator AND}

As we have previously noticed, one of the advantages of bitmap arrays is that we may use some logical operators on them while managing rules. For example, we may use the logical operator AND. The result of applying an AND between two bitmap arrays is a bitmap array that contains a value ' 1 ' for the item at the index $i$ if the two bitmap arrays contain a '1' for the item at the index $i$.

Thus, using the rules of the previous example, RightPart of R AND RightPart of R' returns the following array:

\begin{tabular}{|c|c|c|c|c|c|c|}
\hline RightPart of $R$ & 0 & 1 & 1 & 0 & 0 & 0 \\
\hline RightPart of $R^{\prime}$ & 0 & 1 & 1 & 0 & 1 & 0 \\
\hline AND & 0 & 1 & 1 & 0 & 0 & 0 \\
\hline
\end{tabular}

To determine the number of items associated with a bitmap array, we only have to count the number of ' 1 ' in it.

\section{EVALUATING THE NON REDUNDANCY OF ONE RULE}

\subsection{With the basic storage}

If the set of association rules is stored using the classical representation, that is using the single table RULES presented in the previous section, searching for non redundant rules in it leads to traverse the whole table. To determine if a rule $R$ is non redundant with respect to a rule $R^{\prime}$, we have to compare the left parts of $R$ and $R^{\prime}$ and then their right parts. To do so, we have to join the table RULES with itself.

The SQL request used to determine if the left part of a rule $R$ is not included in the left part of a rule $R^{\prime}$ is this one:

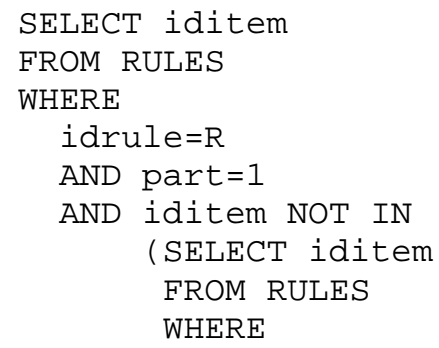

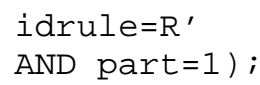

Processing right parts leads to a similar SQL request.

We may observe that the processing time depends on the number of items per rule and on the number of rules in RULES. Furthermore, join operator being time consuming, this first solution does not seem to be the best one and it seems preferable not to do such operations on the RULES which is usually huge.

\subsection{With bitmap arrays}

We show the way we determine the non redundancy of a rule with respect to another rule using the efficient representation of rules based on bitmap arrays. This will allow us, in the next section, to present an algorithm for discovering all the non redundant rules from a rulebase. This algorithm will not reduce the number of tests to be done, but it will allow us to never process any join and consequently to dramatically decrease the processing times. This algorithm makes the most of a property of bitmap arrays with respect to redundancy of rules.

Let $R=B \rightarrow H$ and $R^{\prime}=B^{\prime} \rightarrow H^{\prime}$ be two association rules defined by $B=\left\{B_{1}, \ldots, B_{n}\right\}, H=$ $\left\{H_{1}, \ldots, H_{m}\right\}, B^{\prime}=\left\{B_{1}^{\prime}, \ldots, B_{n^{\prime}}^{\prime}\right\}$ and $H^{\prime}=$ $\left\{H_{1}^{\prime}, \ldots, H_{m^{\prime}}^{\prime}\right\}$ on $I=\{1, \ldots, k\}$.

Let us recall that, in this paper, we consider that an association rule $R=B \rightarrow H$ is non redundant if there exists no rule $R^{\prime}=B^{\prime} \rightarrow H^{\prime}$ such that $B^{\prime} \subseteq B$ and $H \subseteq H^{\prime}$

We call $I B_{X}=\left\{I B_{1}^{X}, \ldots, I B_{k}^{X}\right\}$ the bitmap array corresponding to the left part of the rule $X$ and $I H_{X}=\left\{H_{1}^{X}, \ldots, I H_{k}^{X}\right\}$ the bitmap array corresponding to the right part of the rule $X$ where $I B_{i}^{X}$ is equal to 1 if the item $i$ is contained in the left part of $X, 0$ else, and $I H_{i}^{X}$ is equal to 1 if the item $i$ is contained in the right part of $X, 0$ else.

We note ( $R$ AND $R^{\prime}$ ) the rule having as a left (resp. right) part the intersection of the left (resp. right) parts of the rules $R$ and $R^{\prime}$ :

$$
\left(R A N D R^{\prime}\right)=B \cap B^{\prime} \rightarrow H \cap H^{\prime}
$$

Proposition: The rule $R$ is redundant with respect to the rule $R^{\prime}$ if and only if $I B_{\left(R A N D R^{\prime}\right)}=I B_{R^{\prime}}$ and $I H_{\left(R A N D R^{\prime}\right)}=I H_{R}$.

\section{Proof:}

Let us show that if $R$ is redundant with respect to $R^{\prime}$ then $I B_{\left(R A N D R^{\prime}\right)}=I B_{R^{\prime}}$ and $I H_{\left(R A N D R^{\prime}\right)}=I H_{R}$. Due to the way bitmap arrays are constructed, $I B_{\left(R A N D R^{\prime}\right)}$ is the intersection of the left parts of the rules $R$ and $R^{\prime}$.

So, if $R$ is redundant with respect to $R^{\prime}$ then $I B_{R^{\prime}} \subseteq$ $I B_{R}$.

Thus, $I B_{\left(R \text { AND } R^{\prime}\right)}=I B_{R} \cap I B_{R^{\prime}}=I B_{R^{\prime}}$.

In the same way, $I H_{\left(R A N D R^{\prime}\right)}$ is the intersection of 
the right parts of the rules $R$ and $R^{\prime}$.

So, if $R$ is redundant with respect to $R^{\prime}$ then $I H_{R} \subseteq$ $I H_{R^{\prime}}$.

Thus, $I H_{\left(R \text { AND } R^{\prime}\right)}=I H_{R} \cap I H_{R^{\prime}}=I H_{R}$.

In conclusion, if $R$ is redundant with respect to $R^{\prime}$ then $I B_{\left(R A N D R^{\prime}\right)}=I B_{R^{\prime}}$ and $I H_{\left(R A N D R^{\prime}\right)}=I H_{R}$. Reciprocal:

Let us demonstrate that if $R$ is not redundant with respect to $R^{\prime}$ then $I B_{\left(R A N D R^{\prime}\right)} \neq I B_{R^{\prime}}$ or $I H_{\left(R A N D R^{\prime}\right)} \neq I H_{R}$.

By definition, if $R$ is non redundant with respect to $R^{\prime}$ then $B^{\prime}$ is not included in $B$ or $H$ is not included in $H^{\prime}$.

If $B^{\prime}$ is not included in $B$, then $I B_{R} \cap I B_{R^{\prime}} \neq I B_{R^{\prime}}$. So $I B_{\left(R A N D R^{\prime}\right)}=I B_{R} \cap I B_{R^{\prime}}$. Thus, $I B_{\left(R A N D R^{\prime}\right)} \neq I B_{R^{\prime}}$.

Else, $H$ is not included in $H^{\prime}$, then $I H_{R} \cap I H_{R^{\prime}} \neq I H_{R}$.

So $I H_{R A N D H^{\prime}}=I H_{R} \cap I H_{R^{\prime}}$ and consequently $I B_{\left(R A N D R^{\prime}\right)} \neq I B_{R^{\prime}}$. qed.

Example: If we consider again the two rules given as example in the previous section we may see that $R$ is redundant with respect to $R^{\prime}$. Indeed, the bitmap array LeftPart of ( $R$ AND $R^{\prime}$ ) is equal to (000001):

\begin{tabular}{|c|c|c|c|c|c|c|}
\hline LeftPart of R & 0 & 0 & 0 & 1 & 0 & 1 \\
\hline LeftPart of R' & 0 & 0 & 0 & 0 & 0 & 1 \\
\hline AND & 0 & 0 & 0 & 0 & 0 & 1 \\
\hline
\end{tabular}

The bitmap array LeftPart of ( $R$ AND $R^{\prime}$ ) is equal to the bitmap array RightPart of $R^{\prime}$.

In the same way, the bitmap array RightPart of ( $R$ AND $R^{\prime}$ ) is equal to (011000), indeed:

\begin{tabular}{|c|l|l|l|l|l|l|}
\hline RightPart of R & 0 & 1 & 1 & 0 & 0 & 0 \\
\hline RightPart of R' & 0 & 1 & 1 & 0 & 1 & 0 \\
\hline AND & 0 & 1 & 1 & 0 & 0 & 0 \\
\hline
\end{tabular}

So, the bitmap array RightPart of ( $R$ AND $\left.R^{\prime}\right)$ is equal to the bitmap array RightPart of $R$.

If the right part of $R^{\prime}$ had been $\{\mathrm{B}, \mathrm{E}, \mathrm{F}\}$, then $R$ would not had been redundant with respect to $R^{\prime}$. Indeed, the bitmap array RightPart of ( $R$ AND $R^{\prime}$ ) would have been equal to (010000):

\begin{tabular}{|c|c|c|c|c|c|c|}
\hline RightPart of R & 0 & 1 & 1 & 0 & 0 & 0 \\
\hline RightPart of R' & 1 & 1 & 0 & 0 & 1 & 0 \\
\hline AND & 0 & 1 & 0 & 0 & 0 & 0 \\
\hline
\end{tabular}

So the bitmap array RightPart of ( $R$ AND $R^{\prime}$ ) is not equal to the bitmap array RightPart of $R$.

\section{SEARCHING FOR NON REDUNDANT RULES IN A RULE BASE}

We now present the algorithm 1 designed for searching for non redundant rules in a rulebase and using the property demonstrated in the last section. From a list RuleList containing the set of $N$ rules to be tested, it returns an array NRR of size SizeNRR in which each non redundant rule found by the algorithm is stored.

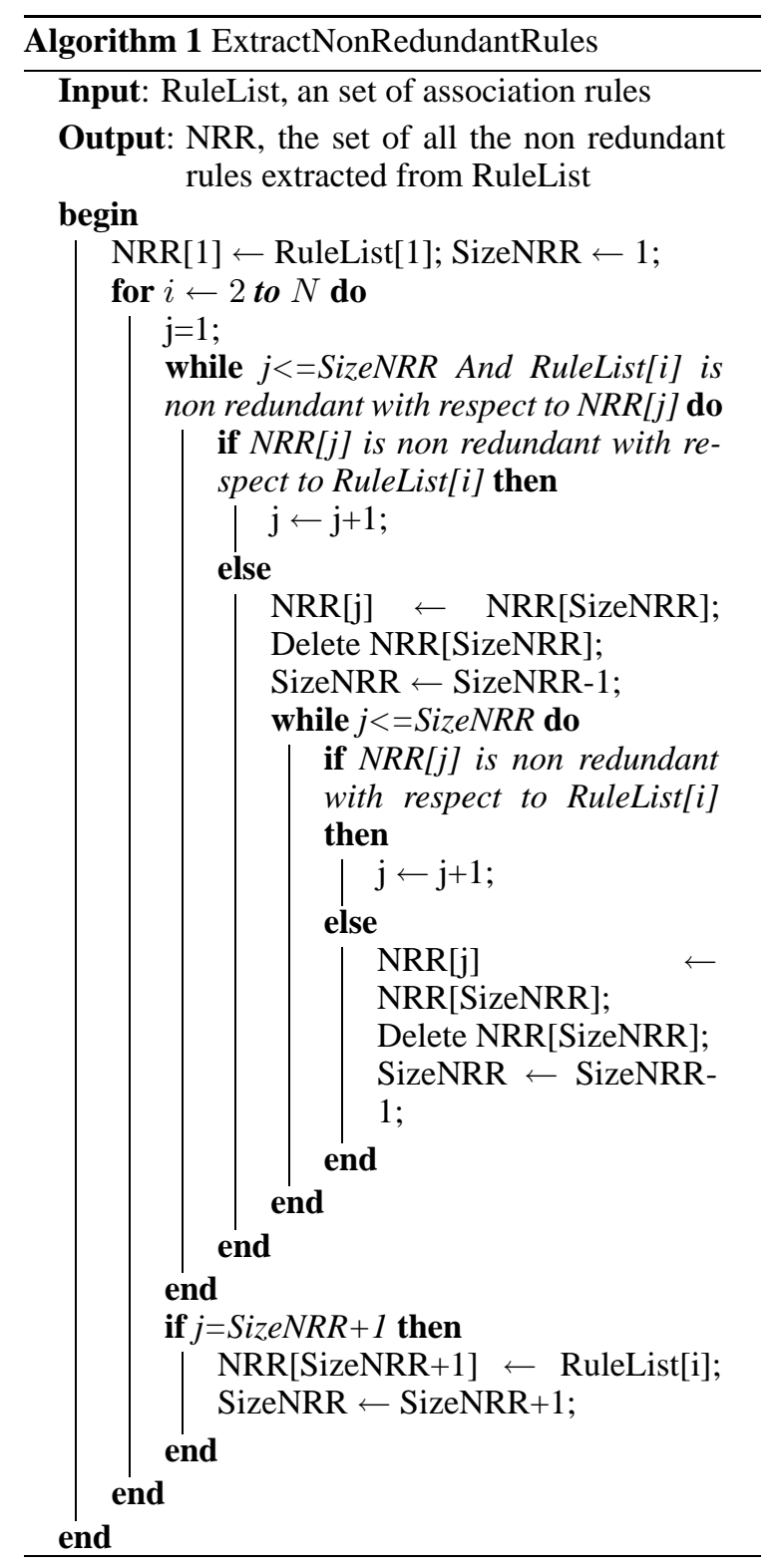

The array $N R R$ is built in such a way that the rules it contains are non redundant the ones against the others. This principle allows us to decrease the number of redundancy tests. Indeed, if a rule $j$ of $N R R$ is redundant with respect to a rule $i$ of RuleList, it is useless to test if the rule $i$ is redundant with respect to the other rules of $N R R$ because it is impossible. Indeed, let us suppose it exists a rule $k$ of $N R R$ such that the rule $i$ was redundant with respect to the rule 
$k$. Then, as the rule $j$ is redundant with respect to the rule $i$, in that case, $B_{i} \subseteq B_{j}$ and $H_{j} \subseteq H_{i}$. Similarly, the rule $i$ being redundant with respect to the rule $k$ we have $B_{k} \subseteq B_{i}$ and $H_{i} \subseteq H_{k}$. Thus, $B_{k} \subseteq B_{j}$ and $H_{j} \subseteq H_{k}$ by transitivity, which means that the rule $j$ of $N R R$ is redundant with respect to the rule $k$ of $N R R$ which contradicts the way we construct $N R R$.

\section{EXPERIMENTS}

We have made several experiments to compare the processing time needed to extract non redundant rules from a set of rules using the basic approach and the bitmap one. In the two cases the global number of rules of the base and the number of non redundant rules it contains may have an influence on the efficiency, thus some experiments have been done varying successively those two parameters. We also have studied the influence of the number of items per rule on the efficiency of the system.

The first experiment (figures 1(a) and 1(b)) studies the influence of the total number of rules in the pattern base on the processing time needed to discover all the non redundant rules. The bitmap array approach is really more efficient than the classical one which is between 20 to 100 times slower. Moreover, even if the processing time increases with the number of rules in the two approaches, the increase is stronger for the classical one. The processing time with the bitmap array approach remains reasonable even for 7000 rules (approximately 4 minutes) while it takes approximately one hour with the classical approach for processing 1000 rules.

The second experiment (figures 2(a) and 2(b)) studies the time needed to discover the non redundant rules, depending on their number. The results are rather encouraging: the bitmap approach is between 37 and 50 times faster than the basic approach and the increase of the processing time with the number of non redundant rules is lower for the bitmap approach than for the basic one.

The last experiment, which studies the influence of the number of items per rule on the processing time 3(a) et 3(b)), confirms the previous results: the basic approach is strongly influenced by the number of items per rule (more than 9 times slower between 8 and 30 items per rule) while the bitmap one is less influenced by that (less than two times slower between 8 and 30 items per rule). In terms of processing time, the bitmap approach is really acceptable (with a maximum of 26 seconds) while the basic one exceeds one hour for processing a rule base where rules contain 30 items.

Finally we have studied the influence of the global

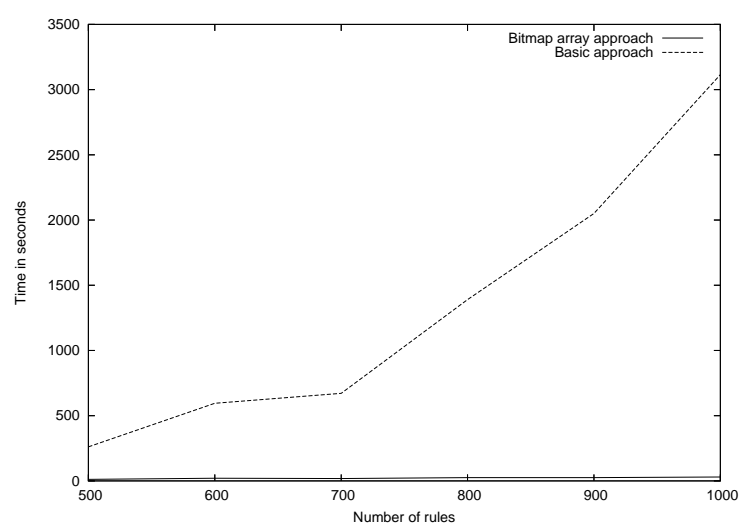

(a)

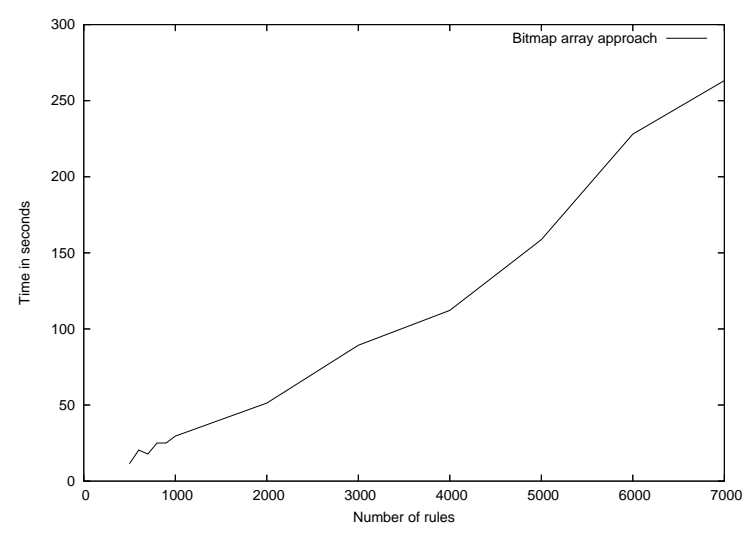

(b)

Figure 1: Processing time depending on the number of rules

number of items used in the whole rulebase. This experiment have shown that the efficiency of the two approaches was similar and that this parameter does not have any influence on it.

\section{CONCLUSION AND FUTURE WORKS}

In view of the large pattern bases that can be generated nowadays by various efficient data mining algorithms, it becomes essential to have efficient tools for pattern management. In this paper, we were interested in association rules and more precisely the rapid discovery of non redundant rules from large rulebases. To do so, we have considered the use of bitmap arrays and the logical operator AND. The experiments 


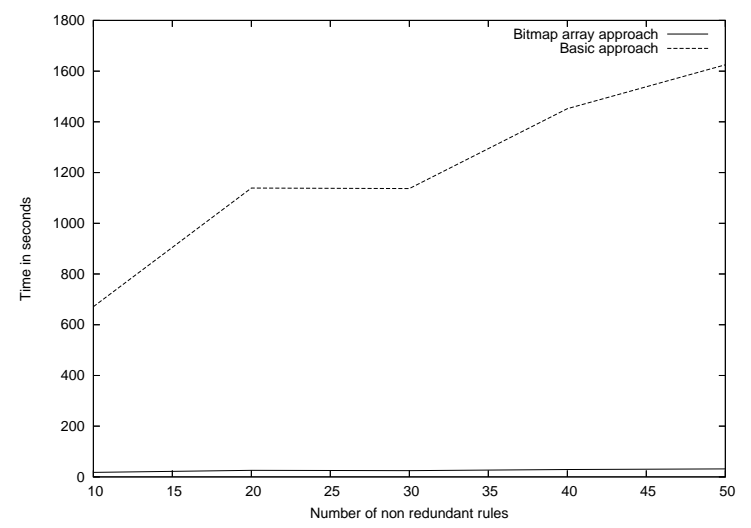

(a)

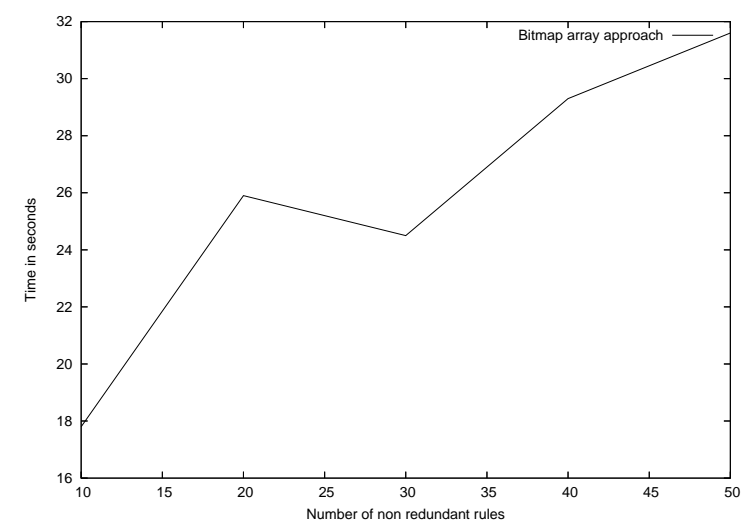

(b)

Figure 2: Processing time depending on the number of non redundant rules to be discovered

we made have shown that this approach is dramatically more efficient than the basic one, allowing us to process large pattern bases in linear time.

Our research aims at designing a more general framework for a pattern base management system and thus we have to develop the work presented here in several ways. For example, using bitmap arrays for coding association rules may allow us to design other rule management operators such as discovering rules that contain a given set of items, or rules that contradict a given hypothesis, etc.

At the same time, we want to investigate the storage and management of other classes of patterns such as clusters, decision trees, etc.

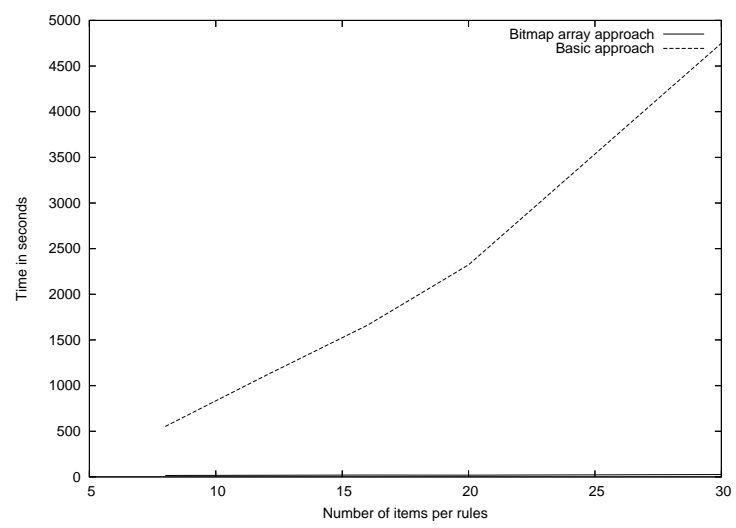

(a)

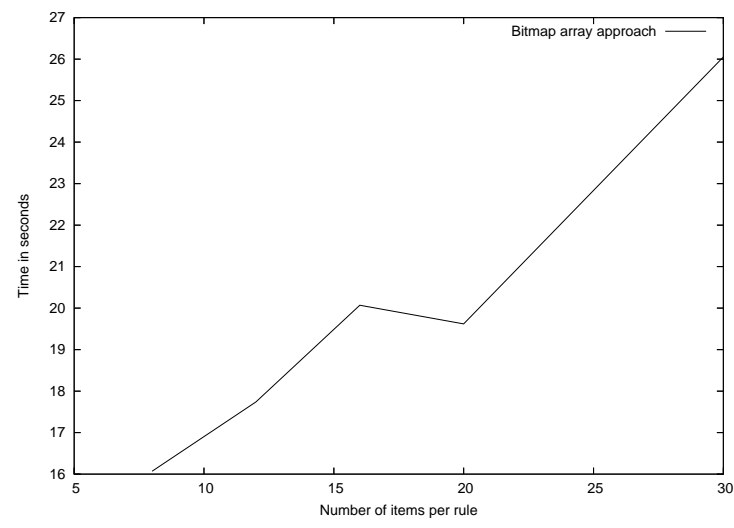

(b)

Figure 3: Processing time depending on the number of items per rule

\section{REFERENCES}

Aggarwal, C. C. and Yu, P. S. (1998). Online generation of association rules. In Proceedings of the 14th Conference on Data Engineering, pages 402-411, Orlando.

Agrawal, R., Imielinski, T., and Swami, A. (1993). Mining association rules between sets of items in large databases. In Proceedings of the ACM SIGMOD International Conference on Management of Data, pages 207-216, Washington D.C.

Albert-Lorincz, H. and Boulicaut, J.-F. (2003). Mining frequent sequential patterns under regular expressions: A highly adaptive strategy for pushing contraints. In Proceedings of the Third SIAM International Conference on Data Mining, San Francisco, CA, USA, May 1-3, 2003.

Bastide, Y., Pasquier, N., Taouil, R., Stumme, G., and Lakhal, L. (2000). Mining minimal non-redundant 
association rules using frequent closed itemsets. In Proceedings of the first International Conference on Computational Logic, LNCS 1861, pages 972-986.

Bayardo, R., Agrawal, R., and Gunopulos, D. (2000). Constraint-based rule mining in large, dense databases. Data Mining and Knowledge Discovery, 4(2/3):217-240.

Boulicaut, J. and Jeudy, B. (2005). Constraint-based data mining. In The Data Mining and Knowledge Discovery Handbook, pages 399-416. Springer.

Boulicaut, J. F. (2005). Condensed representations for data mining. In Encyclopedia of Data Warehousing and Mining, pages 207-211. Idea Group Reference.

Boulicaut, J. F. and Masson, C. (2005). Data mining query languages. In The Data Mining and Knowledge Discovery Handbook, pages 715-727. Springer.

Catania, B., Maddalena, A., Mazza, M., Bertino, E., and Rizzi, S. (2004). A framework for data mining pattern management. In Proceedings of the 8th European Conference on Principles and Practice of Knowledge Discovery in Databases, LNCS 3202, pages 87-98.

Freitas, A. A. (1999). On rule interestingness measures. Knowledge-Based Systems, 12(5-6):309-315.

Garofalakis, M. N., Rastogi, R., and Shim, K. (2002). Mining sequential patterns with regular expression constraints. IEEE Transactions on Knowledge and Data Engineering, 14(3):530-552.

Goethals, B., Muhonen, J., and Toivonen, H. (2005). Mining non-derivable association rules. In Proceedings of the fifth SIAM International Conference on Data Mining, Newport Beach, California, USA, April 21-23.

Grossman, R. L., Bailey, S., Ramu, A., Malhi, B., Hallstrom, P., Pulleyn, I., and Qin, X. (1999). The management and mining of multiple predictive models using the predictive model markup language (PMML). In Information and Software Technology, volume 41, pages 589-595.

Li, G. and Hamilton, H. (2004). Basic association rules. In Proceedings of the fourth SIAM International Conference on Data Mining, Lake Buena Vista, Florida, USA, April 22-24. SIAM.

Li, Y., Liu, Z. T., Chen, L., Cheng, W., and Xie, C. H. (2004). Extracting minimal non-redundant association rules from QCIL. In International Conference on Computer and Information Technology, pages 986991. IEEE Computer Society.

Louie, E. and Lin, T. Y. (2000). Finding association rules using fast bit computation: Machine-oriented modeling. In Proceedings of the 12th International Symposium on Methodologies for Intelligent Systems, LNCS 1932, pages 486-494. Springer.

Masson, C., Robardet, C., and Boulicaut, J. F. (2004). Optimizing subset queries: a step towards sql-based inductive databases for itemsets. In Proceedings of the 2004 ACM symposium on Applied computing (SAC'04), pages 535-539. ACM Press.
Morzy, T. and Zakrzewicz, M. (1998). Group bitmap index: A structure for association rules retrieval. In Proceedings of the Fourth International Conference on Knowledge Discovery and Data Mining, pages 284288. AAAI Press.

Pasquier, N., Taouil, R., Bastide, Y., Stumme, G., and Lakhal, L. (2005). Generating a condensed representation for association rules. Journal of Intelligent Information Systems, 24(1):29-60.

Pucheral, P., Gardarin, G., and Wu, L. (1998). Bitmap based algorithms for mining association rules. In Actes des Journées Bases de Données Avancées (BDA'98).

Tuzhilin, A. and Liu, B. (2002). Querying multiple sets of discovered rules. In Proceedings of the Eighth ACM SIGKDD International Conference on Knowledge Discovery and Data Mining, pages 52-60. ACM.

Wang, J. and Han, J. (2004). BIDE: Efficient mining of frequent closed sequences. In Proceedings of the 20th International Conference on Data Engineering, ICDE 2004, 30 March - 2 April 2004, Boston, MA, USA, pages 79-90.

Wang, J., Han, J., Lu, Y., and Tzvetkov, P. (2005). TFP: An efficient algorithm for mining top-k frequent closed itemsets. IEEE Transactions on Knowledge and Data Engineering, 17(5):652-664.

Zaki, M. J. (2000). Generating non-redundant association rules. In Proceedings of the sixth ACM SIGKDD international conference on Knowledge discovery and data mining, August 20-23, 2000, Boston, MA, USA, pages 34-43.

Zaki, M. J. (2001). SPADE: An efficient algorithm for mining frequent sequences. Machine Learning, 42(1/2):31-60.

Zaki, M. J. (2004). Mining non-redundant association rules. Data Mining and Knowledge Discovery, 9(3):223248.

Zaki, M. J. and Hsiao, C. (2005). Efficient algorithms for mining closed itemsets and their lattice structure. IEEE Transactions on Knowledge and Data Engineering, 17(4):462-478.

Zaki, M. J., Parimi, N., De, N., Gao, F., Phoophakdee, B., Urban, J., Chaoji, V., Hasan, M. A., and Salem, S. (2005). Towards generic pattern mining. In Proceedings of the Third International Conference on Formal Concept Analysis, pages 1-20. 